\title{
CRISIS DE LA ENSEÑANZA DE LA GEOMETRÍA
}

FERNANDO JORQUERA MOLINA

Existe una impresión general, que la enseñanza de la geometría, ha sufrido un deterioro en estos últimos años. Análisis realizados el año pasado en la Universidad de Santiago así lo confirman.

E1 primer estudio se refiere a los resultados de una prueba de diagnóstico, sobre contendidos de Geometría del Programa Oficial, administrada a los alumnos de la Carrera de Licenciatura en Educación Matemática de la U. de Santiago, que ingresaron en el 2 Semestre de 1981 La prueba, se construyó en base a 139 ítems de selección múltiple, que se redactaron de acuerdo a los objetivos elaborados para cada uno de los con tenidos del programa de $1^{\circ}$ a $3^{\circ}$ medio.

La muestra estaba compuesta por 173 alumnos, con un promedio de 620 puntos en la P.A.A. A pesar de 
que estos alumnos debieran estar mejor preparados en matemática que el común de los egresados de enseñanza media, por postular a una carrera Matemática, el resultado fue muy inferior a lo esperado. E1 $80 \%$ de los objetivos medidos no se dominaban o semidominaban y sólo un $20 \%$ de 1 os objetivos fue aprobado. Conceptos como: Trazo dirigido, Transformaciones geométricas, Paralelismo, Distancia entre dos puntos, etc., se desconocían, y se demostraba cierto conocimiento en tópicos como Teorema de Thales, Teorema de Pitágoras, Semejanza, Volumen de un paralelepípedo.

E1 segundo estudio, se refiere a un test administrado a 554 alumnos pertenecientes a 19 Liceos del Area Metropolitana, eligiéndose en cada uno de ellos un curso de $4^{\circ}$ medio al azar.

E1 resultado de esta prueba fue aún más bajo, demostrando los alumnos un total desconocimiento de los tópicos geométricos. Un solo objetivo, el que se refería al Teorema de Pitágoras, fue alcanzado por sobre el $70 \%$ de los alumnos de esta muestra. Este estudio reveló además, que más del $50 \%$ de los contenidos indicados en el Programa Oficial, no son tratados por los profesores.

E1 tercer estudio que nos muestra también la crisis en el conocimiento de los contenidos geométricos, está basada en un estudio realizado por el Profesor Wadin Praus Petroff de la Universidad Católica de Valparaíso. Comprende 5 aplicaciones de la P.C.E. en Matemática de los años 1975 a 1979 inclusive. Estas pruebas constaron de 229 itemes de los cuales 39 de ellos correspondían a geometría. La cantidad de alumnos que rindieron dichas pruebas fue de 399.475. El puntaje medio general, fué de 556 puntos. En esta muestra, también se verifica el bajo rendimiento en geometría, ya que de 39 itemes, hay un porcentaje medio de 
$25,5 \%$ de conocimiento, resulta por tanto un $74,5 \%$ de No Dominio.

Otro indicador sobre el deterioro de la enseñanza de la Geometría en la Enseñanza Media, es observar la disminución de los contenidos de geometría a través de los diferentes programas de Matemática que ha tenido la Educación Chilena.

Podemos comprobar que desde 1908, a la fecha se han decretado 12 programas en que se indican contenidos matemáticos. En los primeros programas (de 1908, 1912, 1929) se indicaba las horas de aritmética, álgebra y geometría por separado. Los programas hasta el año 1955 contenían bastante geometría; a partir de ler. Año de Humanidades se introducía el alumno, en la Geometría Euclideana y en la Geometría del Espacio; se estudiaban las por piedades de los Triángulos y Cuadriláteros, Construcciones Geométricas, Lugares Geométricos, la Circunferencia, Semejanza, Simetrías, Rotaciones, Traslaciones y Homotecias, Cuerpos Geométricos, Cálculo de Areas y Volúmenes.

Con la reforma de 1968, se creó la Educación Básica con 8 años y la Educación Media con 4 años. Los nuevos Programas de Matemática quisieron recoger entonces, las orientaciones de la Matemática Moderna, dándole importancia a la estructuras.

A partir de estos programas la geometría fue perdiendo importancia, ya que la Geometría Clásica quedó en programas de Educación Básica. En Educación Media los programas de Matemática contenían Geometría sólo en ler. y 2do. Años medios y siguiendo las orientaciones modernas, se incluyó Geometría Vectorial, Geometría dirigida y de Trans- 
formaciones. Pero, al no estar los profesores preparados, ya que la implementación de estos programas fue deficiente (no se entregaron orientaciones básicas, material de apoyo textos, ni cursos) no se enseñó ni la antigua, ni la nueva geometría.

A partir del Programa de 1968, se efectuaron varias reformas en los años 74, 76 y 78. Si el Programa de 1968, tenía una clara orientación de Geometría Moderna, las modificaciones efectuadas en los Programas posteriores 10 desfiguran totalmente obteniéndose un conjunto de contenidos sin relación alguna entre sí; una confusión entre Geometría Afín, Geometría Vectorial, Geometría Métrica, Geometría Analítica, confundiendo por ejemplo un vector con un par ordenado de reales y con un punto euclidiano. Este hecho hizo más difícil la enseñanza de la Geometría.

La realidad del bajo conocimiento geométrico de los egresados de Enseñanza Media y la pobreza de los Programas en contenidos geométricos, nos plantean un grave problema, dada la importancia que ella tiene en la formación integral del individuo.

Muchas son las razones que justifican la inclusión de contenidos geométricos en los Programas de Enseñanza Media y en los Programas de Matemáticas para la formación de algunos profesionales. Influido tal vez por mi condición de Educador, los argumentos que aquí ocuparé, tienen que ver más bien con la formación integral del individuo, considerando que el objetivo final de la Matemática en las Sociedades Modernas, es enseñar a los educandos a ordenar y encadenar sus pensamientos con arreglo al método que emplea la matemática, desarrollar la creatividad, el espíritu crítico, la imaginación y el espíritu de observación. 
Educar es, desde Aristóteles, el paso de la potencia al acto; la educación es fundamentalmente una conquista personal y para adquirir esta conquista, en Matemática, no basta con estudiarla, ni siquiera aprenderla, sino, hay que hacerla. Es este quehacer el que posibilitará al alumno para desenvolverse acertadamente en el futuro, que en la Sociedad actual en acelerado proceso de cambio, se nos muestra imprevisible. Mirando a ese futuro, importan más las actitudes y aptitudes que los conocimientos.

Los sicólogos distinguen entre una conducta aprendida y una conducta inteligente que habilita al que la posee para reaccionar, previo análisis de los factores que la integran, gracias a su actitud discursiva, ante una situación nueva. Para mí esta capacidad de adaptación y reacción ante una situación nieva es objetivo fundamental de la Educación de hoy.

En este planteamiento es, a mi juicio, en el que la geometría - la más antigua de las ciencias- sigue teniendo plena vigencia. Su riqueza de situaciones ofrece al alumno la posibilidad de ejercitarse en el desarrollo de su intuición creadora y en la formulación de conjeturas posibles, así como, en un estudio más avanzado, en la adquisición de hábitos correctos de pensar y en la comprobación de la validez de un razonamiento deductivo.

En otras palabras mi argumento se puede sintetizar en los siguientes puntos:

1. La Geometría es la más antigua de las ramas de la Matemática y la primera en ocupar el método deductivo. Repitiendo una frase de Klein: Se deberá conducir a 
la juventud teniendo en cuenta su natural capacidad y disposición, lentamente hasta llegar a las nociones elevadas siguiendo el mismo camino por el que la humanidad ha ascendido desde un estado primitivo a las altas cumbres del conocimiento científico" . Por eso, de acuerdo al método histórico, los contenidos de tipo geométricos no pueden estar ausentes de un currículum de enseñanza elemental.

2. La rapidez en tomar decisiones del individuo en el mundo actual, obliga a formarlos con una claridad mental que les permita decidir con facilidad la alternativa adecuada en el quehacer de su vida.

Aquí el método de la Geometría, adiestra mejor que ninguna otra asignatura en la capacidad de seguir razonamientos y obtener conclusiones de acuerdo a dicho razonamiento.

3. Creo que los contenidos y en la presentación de ellos, esta asignatura es la que mejor se presta para desarrollar en el educando la intuición creadora y formar la imaginación. Los problemas de construcciones y de lugares geométricos, son os mejores ejercicios que permiten hacer crecer dichas capacidades.

4. A nadie escapa también el valor práctico que tienen algunos contenidos geométricos, básicos para la comprensión de michos conceptos matemáticos. Una buena base en Geometría permite más fácilmente el avance en la adquisición de nuevos conocimientos matemáticos.

5. En estos momentos el paso de la enseñanza media a la universitaria, presenta varias dificultades de adaptación a nuestros estudiantes, que se traduce en un al- 
to porcentaje de reprobación en los primeros cursos de matemática en la Universidad. Pienso que una mejora base geométrica permitiría una más adecuada adaptación.

En definitiva, mi tesis es que existe un gran deterioro de la Enseñanza de la Geometría en la Enseñanza Media y en los Cursos de Matemática de la Universidad. Este hecho va a significar a un corto plazo una baja en el nivel de conocimientos matemáticos en el país.

Sostengo que debe conservarse en los programas de matemática, una parte importante destinada a la "Geometría tradicional", si bien, modernizada en su tratamiento metodológico con un nuevo enfoque en la manera de presentarla. 